\title{
CORRECTION
}

\section{Correction to: Teen suicide: an underreported and emerging plague}

\author{
Emiliano Gamberini ${ }^{1} \cdot$ Etrusca Brogi ${ }^{1}$ (C) Domenico Pietro Santonastaso ${ }^{1} \cdot$ Emanuele Russo $^{1} \cdot$ Vanni Agnoletti $^{1}$. \\ Costanza Martino ${ }^{1}$
}

Published online: 19 December 2020

(c) Società Italiana di Medicina Interna (SIMI) 2021

\section{Correction to: Internal and Emergency Medicine https://doi.org/10.1007/s11739-020-02554-2}

In the original publication of the article, the 3rd author surname and the 6th author given name was incorrectly published. The correct author name is given in this erratum.

The original article was corrected.

Publisher's Note Springer Nature remains neutral with regard to jurisdictional claims in published maps and institutional affiliations.

The original article can be found online at https://doi.org/10.1007/ s11739-020-02554-2.

Etrusca Brogi

etruscabrogi@gmail.com

Emiliano Gamberini

emiliano.gamberini@auslromagna.it

Domenico Pietro Santonastaso

domenicopietro.santoanastaso@auslromagna.it

Emanuele Russo

emanuele.russo@auslromagna.it

Vanni Agnoletti

vanni.agnoletti@auslromagna.it

Costanza Martino

costmartino74@gmail.com

1 Department of Anesthesia and Intensive Care, Bufalini Hospital, Viale Giovanni Ghirotti, 286, 47521 Cesena FC, Italy 